\title{
Sphere-to-Plate Glass Welding using Picosecond-Laser Radiation
}

\author{
Karolis RATAUTAS, Gediminas RAČIUKAITIS, Mindaugas GEDVILAS \\ Center for Physical Sciences and Technology, Savanoriu Ave. 231, Vilnius, LT-02300, Lithuania \\ E-mail: graciukaitis@ar.fi.lt
}

\begin{abstract}
Welding of a spherical shaped glass beads with the diameter of $0.7 \mathrm{~mm}$ to a float glass plate by using picosecond laser irradiation was investigated. The glass bead itself acts as focusing lens and should be considered as a part of optical system. The different number of pulses, repetition rate and average laser power were applied to estimate the joint strength dependence on the laser process parameters. Modeling of transient thermal fields induced by laser irradiation was performed in order to evaluate a possible defect formation. Two types of crack-free welding regimes were found, namely, the point welding with a low number of laser pulses and a weak joint and the fusion welding when irradiation lasts up to one second. The joint area is significantly larger and the glass bead can withstand higher compression stress which is important for application in vacuum isolated glasses. The processes still need to be optimized in terms of productivity.
\end{abstract}

DOI:10.2961/jlmn.2013.02.0011

Keywords: glass welding, picosecond laser, vacuum isolated glass

\section{Introduction}

Glass is a widely-used material in many areas of technology and industry, from optics to building construction [1]. Plenty application fields require precise joining techniques of the glass. Laser welding of glass materials can be one of the easiest and fastest ways to produce a glass-toglass joint. The glass joining by welding has a huge advantage - it does not pollute the joint with undesirable material (glue, etc.).

Increasing prices on energetic resources and demands on ,green“ environment stimulates to paying a lot of attention to economic use of energy. Saving of energy and preserving of natural resources is especially relevant for residual heating. High thermal isolation of walls and glassing as well is essential way to save large amount of energy on heating of living space. To design energy-efficient buildings, it is essential that all parts of the building envelope have good thermal performance providing effective insulation between the controlled indoor environment and the variable external environment [2]. Thermal losses through walls, characterized by $\mathrm{U}$-value, can be as low as $\mathrm{U}=0.15$ $\mathrm{W} /\left(\mathrm{m}^{2} \mathrm{~K}\right)$. However, windows constitute a weak link in building with the resistance to the heat flow in the best case equal to $\mathrm{U}=1.2 \mathrm{~W} /\left(\mathrm{m}^{2} \mathrm{~K}\right)$ for a double glazing and $0.8 \mathrm{~W} /\left(\mathrm{m}^{2} \mathrm{~K}\right)$ for a triple glazing filled by gasses [3].

Heat transfer through glazing systems occurs in three ways, conduction, convection and radiation [2]. The first two of them could be reduced by evacuation the gap between glasses. Vacuum is the best thermal insulator. The glass block made of two sheets of glass separated by a hermetic gap which has been evacuated is called vacuum insulated glass (VIG). VIG possess the lowest heat conductivity $\left(<0.5 \mathrm{~W} / \mathrm{m}^{2} \mathrm{~K}\right)$ while preserving high transmittance to solar light in infrared spectral rage $(>60 \%)$. A cavity pressure on the order of $\sim 10^{-2} \mathrm{~Pa}$ is required to enable a two to five times better insulation performance compared to the state of the art double glazing even keeping much smaller gap below $1 \mathrm{~mm}[3]$.
Development of VIG production technology is one of the most important tasks in order to implement the concept of the passive house which does not require additional heating. Distance between glass sheets is very important for VIG insulating properties as well as long-term stability of the product. When producing vacuum insulated glass it is necessary to precisely distribute and attach to the glass sheet up to 1000 spacers per $1 \mathrm{~m}^{2}$, which will ensure even and stable distance between glass sheets after evacuation until high vacuum (up to $10^{-4} \mathrm{~Pa}$ ). Insulation and optical properties of the spacers are also very important for VIG product characteristics. To avoid thermal losses, the contact point should be as small as possible. Therefore, a laser welding of the spacers made of glass spheres for their fixture on substrate was proposed in this work.

Glass is a transparent material in the visible spectral range. Successful laser welding by linear absorption was demonstrated only for fused silica using a $\mathrm{CO}_{2}$-laser [4]. Other glass types have a significantly larger thermal expansion coefficient which leads to the development of cracks during the cooling period.

Interest in the glass welding with the ultra-short pulsed lasers working in femtosecond $[5,6]$ and picosecond $[1,7,8$, 9] range increased since the work of Tamaki et. al. in 2005 [10]. The ultra-short laser pulses, at a wavelength transparent to the processing material, are tightly focused onto the joint surface of two glass pieces intended for welding. The fusion welding of glass is achieved by localized heating due to nonlinear light absorption $[7,9,11,12,13]$. The high concentration of electrons can be reached rapidly, initiating avalanche ionization and plasma generation within transparent materials which is able to heat the surrounding material. To keep heating between laser pulses, the highrepetition rate (100s of $\mathrm{kHz})$ lasers are employed. This reduces thermal cycling and effectively suppresses development of thermally induced cracks.

Welding of a spherical shaped bead with the diameter of $0.7 \mathrm{~mm}$ to a float glass plate was investigated. The glass 
bead itself acts as focusing lens. The different number of pulses, repetition rate and average laser power were applied to estimate the joint strength dependence on the laser process parameters. Modeling of transient thermal fields induced by laser irradiation was performed in order to evaluate possible defect formations.

\section{Experimental}

Picosecond laser (Atlantic, Ekspla), with the pulse duration of $10 \mathrm{ps,} \mathrm{was} \mathrm{used} \mathrm{in} \mathrm{experiments.} \mathrm{The} \mathrm{welding} \mathrm{was}$ tested when laser was running at $100 \mathrm{kHz}$ and $500 \mathrm{kHz}$ pulse repetition rate.

Glass sphere acts as a lens and collimated laser beam can be focused with the sphere. However the focus is at the distance roughly equal to its radius beneath the bead (Fig.1a). Glass-to-glass welding with ultra-short pulsed lasers requires localized heating close (tens of micrometers) to the interface between glasses. The collimated laser beam cannot be directly used for welding and combined focusing with the aspheric lens, and, therefore, the glass bead itself was applied (Fig.1b). Such arrangement permitted to control the focal position relative to the beadsubstrate interface. The drawback is that laser beam should be centered relative the sphere.



a)

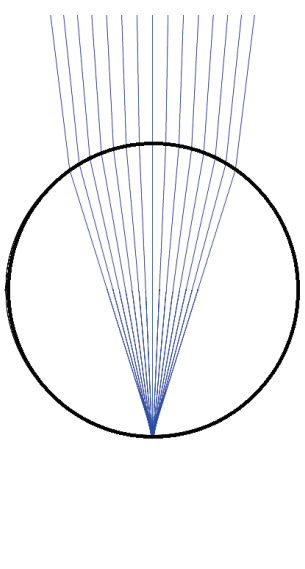

b)
Fig. 1 Focusing of collimated (a) and pre-focused (b) laser beams by a spherical glass bead.

Using combined focusing by a lens and the bead, the exact focal position depends on numerical aperture of the beam incident onto sphere. In order to find the right lens position for focusing to the interface between the bead and the substrate, the shift $\Delta z$ of the focusing position, which appears due to focusing of the laser beam by a bead, needs to be estimated. The shift $\Delta \mathrm{z}$ is a distance that is required to move the focusing lens closer to the glass surface in order to get focusing at the interface between the bead and the substrate. The shift is measured relative to the focusing position without the bead on the substrate surface, as it is show in Fig 2. Experimentally, the exact lens position was found by focusing to the surface of the glass substrate without the bead. After entering the bead into beam paths the objective was displaced by $\Delta z$ and as a result the focus position appeared at the interface of the substrate - bead.

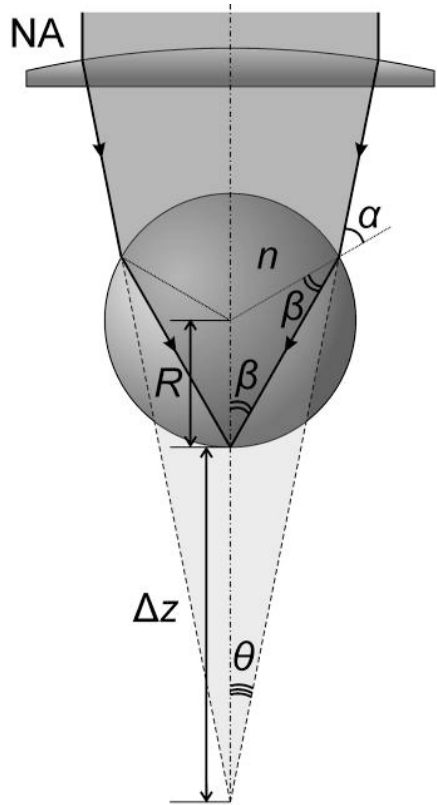

Fig. 2 Scheme of geometrical ray propagation inside the glass bead.

The incident angle of the beam $\theta$ is related to the numerical aperture of the lens NA.

$$
\theta=\arcsin (\mathrm{NA})
$$

According to the Snell's law, the light beam experience refraction by entering media with refraction index $n$ :

$$
\sin \alpha=n \sin \beta
$$

where $\alpha$ is the incidence angle and $\beta$ is the angle of the refracted beam inside the bead.

Geometrical manipulations lead to an equation for a shift of the focal position depending on the incident angle:

$$
\Delta z=R(\sin 2 \beta \cdot \cot \theta-1-\cos 2 \beta)
$$

where $R$ is a radius of the sphere, $\theta=2 \beta-\alpha$ is the angle of incidence without the glass bead. The simulation results according to Eq. (3) on focal position shift are presented in Fig. 3.

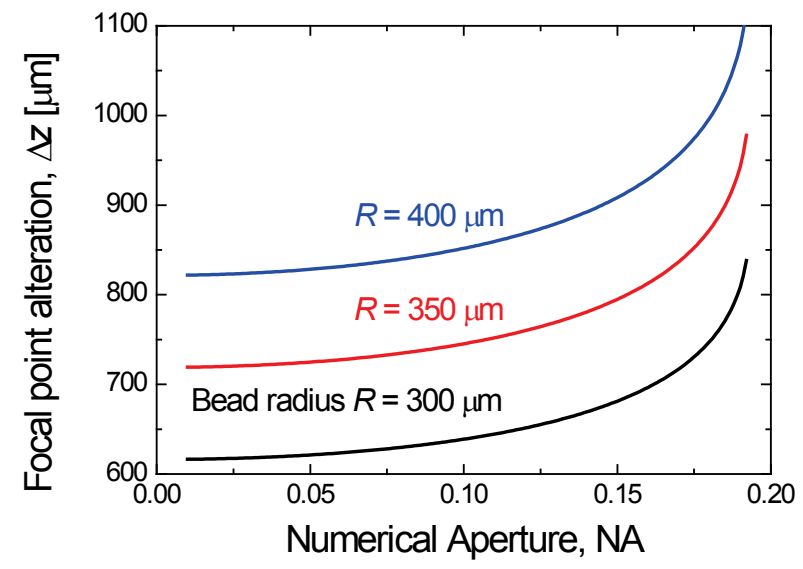

Fig. 3 Focal point alteration as a function of numerical aperture of the focusing lens due to introduction of the glass bead in the beam path. Simulation was made for glass beads with three different sizes. 
Laser beam in experiments was focused with the aspheric lens of the $15.36 \mathrm{~mm}$ focal length (C-260TM, Thorlabs) to the region of the contact point of the glass bead to substrate, as shown in Fig. 4.

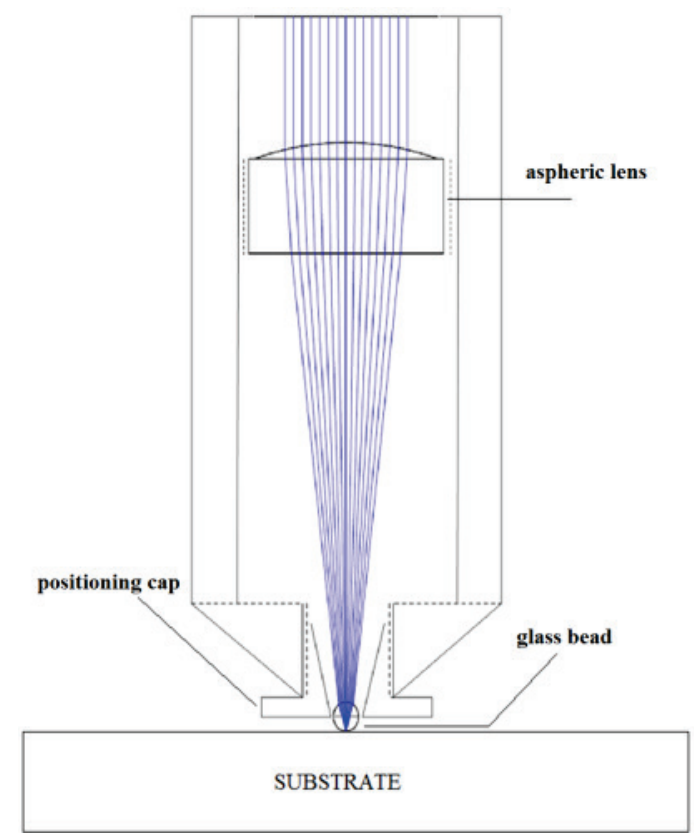

Fig. 4 Focusing head used in experiments.

Experiments were performed on the float glass used for windows glazing (soda-lime-silica glass) and the polished glass spheres made of soda lime glass (SiLibeads, Sigmund Lindner $\mathrm{GmbH}$ ). Thickness of the glass substrate was $4 \mathrm{~mm}$ and diameter of the glass beads was $0.7 \pm 0.15 \mathrm{~mm}$.

Table 1 Physical parameters of glass bead and plate.

\begin{tabular}{lcc}
\hline Parameter & Float glass & Glass bead \\
\hline Young's modulus. GPa & 70 & 65 \\
Poisson coefficient & 0.23 & 0.22 \\
Thermal expansion coeffi- & 9.10 & 8.6 \\
cient, $10^{-6} \mathrm{~K}^{-1}$ & & \\
Tension strength, MPa & $20-100$ & 50 \\
Compressive strength, MPa & 800 & 800 \\
Density, $\mathrm{kg} / \mathrm{m}^{3}$ & 2500 & 2530 \\
Heat capacity, J/(kg K) & 720 & 700 \\
Heat conductivity, W/(m K) & 1.0 & 1.04 \\
Melting temperature, K & 1470 & 1475 \\
\hline
\end{tabular}

Joint between glasses can be produced only if optical contact is achieved. Therefore, the quality of the welded joint depended strongly on a precise positioning of the bead under laser beam. Positioning of the bead in lateral direction relative to the center of laser beam was passively retained by the positioning cap with inner diameter of $0.8 \mathrm{~mm}$

\section{Results}

The glass bead welding was performed by focusing a laser beam through the bead to the contact point with the glass substrate. The welding joint quality was investigated depending on the laser beam average power, the number of pulses, the pulse repetition rate and beam focus position.

\subsection{Thermal modeling}

Thermal model of glass of irradiation was made to determinate transient temperature distribution in welding region. Soda lime and borosilicate glasses are transparent for $1064 \mathrm{~nm}$ length laser wavelength. The linear absorption in such glasses is negligible and is not taken in to account. Welding with $1064 \mathrm{~nm}$ laser light is achieved by focused ultrashort laser beam to a tight spot. Short laser pulse of 10 picoseconds has the high peak energy. Under such condition when a beam is focused to a tight spot, high intensity is achieved and multiphoton absorption takes place. Glass has the band gap $\sim 4.28 \mathrm{eV}$. For the laser wavelength of $1064 \mathrm{~nm}$, photon energy is $1.16 \mathrm{eV}$ and electron must absorb at least four photons in order to be exited to the conduction band.

We used COMSOL Multiphysics thermal conduction model with finite element analysis, to evaluated transient temperature variation during irradiation and at the time between pulses. General heat conduction equation is shown in Eq. (4).

$$
\rho C \frac{\partial T}{\partial t}=\nabla(\kappa \nabla T)+q
$$

where $\rho$ is the glass density, $C$ is the heat capacity, $\kappa$ is the glass thermal conductivity and $q$ represents a heat source, which describes the amount of energy gained by absorbing laser pulse per unit volume and time. The amount of absorbed laser energy with its spatial and temporal distribution inside the glass was simulated taking into account the Gaussian beam propagation close to focal point as well as non-linear absorption. The calculations were performed for the time span covering one laser pulse and the distance between two pulses. Gaussian beam distribution has axial symmetry and it is comfortable to use cylindrical coordinate system. The heat source $q$ was expressed by as follows:

$$
q=\gamma I(z, r)^{4}
$$

here $I(z, r)$ is the laser intensity distribution and $\gamma$ is the four-photon absorption coefficient. The absorption rate is derived from nonlinear absorption differential equation (Beers Law) shown in Eq. (6)

$$
\frac{d I}{d z}=-\alpha_{\mathrm{abs}} I-\gamma I^{4}
$$

Here $\alpha_{\mathrm{abs}}$ is the linear absorption coefficient. Linear absorption is neglected and Eq. (6) could be expressed in simpler expression (7)

$$
\frac{d I}{d z}=-\gamma I^{4}
$$

After calculating the differential Eq. (7) we derivate the absorption rate expressed in Eq. (8)

$$
I(z, r)=\frac{1}{\sqrt[3]{3 \gamma z+\frac{1}{I^{3}}}}
$$

Here $I$ is Gaussian laser pulse (time average) intensity distribution

$$
I(z, r)=I_{0}\left(\frac{\omega_{0}}{\omega_{z}}\right)^{2} \exp \left(\frac{-2 r^{2}}{\omega_{z}^{2}}\right)
$$


Here $\omega_{0}$ is the beam waist and $\omega_{z}$ is the beam radius at distance $z$ from the focus. $I_{0}$ is the laser intensity expressed by Eq. (10) and $\omega_{z}$ by Eq. (11):

$$
\begin{aligned}
& I_{0}=\frac{2 E_{p}}{\tau \pi \omega_{0}^{2}} \\
& \omega_{z}=\omega_{0} \sqrt{1+\left(\frac{z}{z_{r}}\right)^{2}}, \text { where } z_{r}=\frac{\omega_{0}^{2} \pi}{\lambda}
\end{aligned}
$$

$E_{\mathrm{p}}$ is the pulse energy and $\tau$ is the pulse duration.

Temperature distribution in the glass bead after the end of the the 30000 th laser pulse (all with pulse energy of $26 \mu \mathrm{J})$ is shown in Fig. 5.

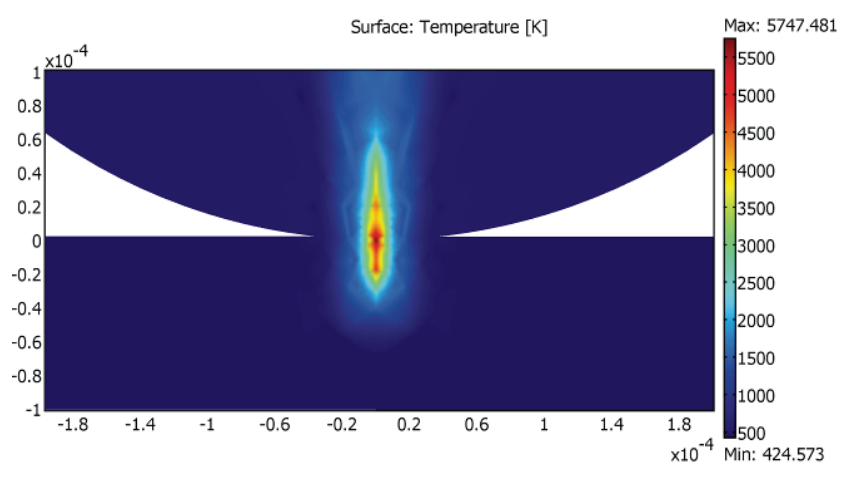

Fig. 5 Temperature distribution at glass-bead interface after irradiation with 30000 laser pulses at $500 \mathrm{kHz}$ repetition rate (60 ms exposure) and $13 \mathrm{~W}$ mean laser power.

\subsection{Bead-to-glass welding @ $100 \mathrm{kHz}$}

Initial welding experiments were performed using the $100 \mathrm{kHz}$ laser pulse repetition rate. Focal point position at the interface of the substrate and the bead was estimated by simulation with ZEMAX. Mean laser power of $5.15 \mathrm{~W}$ $(51.5 \mu \mathrm{J}$ pulse energy) was used to irradiate the contact point between the bead and substrate. Irradiation dose was varies by the number of pulses, ranged from 100 to 300000 . The glass bead started to adhere to the substrate after first 300 laser pulses.

One of the factors which determinated the quality of the welded glass joint was the defects-free seam. Defects that appeared in a bead after welding were cracks in the welding region. The cracks stretched from several microns up to the length over the whole glass bead diameter. As a result showed glass beads irradiated with $5.15 \mathrm{~W}$ average power, developed cracks even after shorter irradiation time of 300 pulses. Thermal modeling with Comsol Multiphysics showed that even after the first pulse irradiation with pulse energy of $51.5 \mu \mathrm{J}$ thermal stresses, which exceeded the glass strength, appeared. Stress in the absorption volume reached $46 \mathrm{MPa}$.

Welded glass beads were observed trough the substrate with optical microscope OLYMPUS. Defects appeared in all beads after irradiation with the $5.15 \mathrm{~W}$ mean laser power. These defects consisted of cracks near to the laser light absorption zone. Image of a crack is shown in Fig. 6.

The length of cracks was measured through the optical microscope. Cracks length depending on the number of laser pulses is shown in Fig. 7. The interesting fact in Fig. 7 is, that if irradiation process stops low number of laser pulses, long cracks develop in the beads. Applying 10000 and more laser pulses, reduces remaining cracks in the beads, probably, due to increased temperature of glass which makes glass ductile close to fusion area.

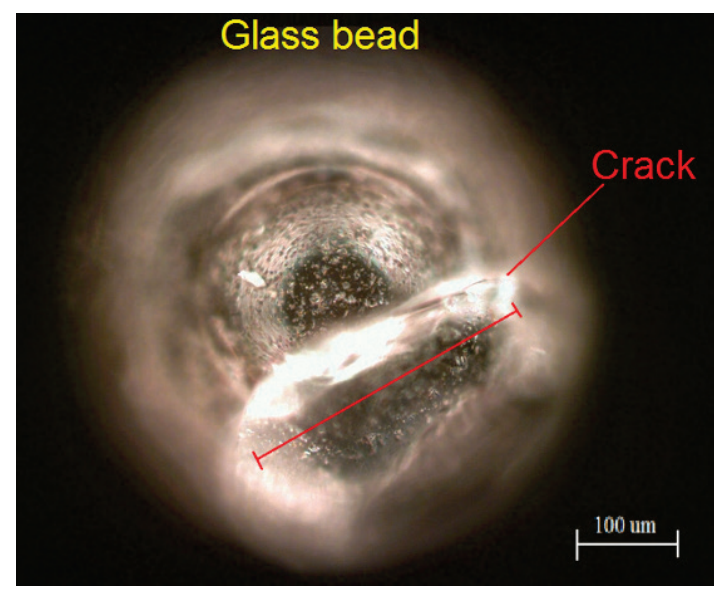

Fig. 6 Crack in the glass bead after 10000 pulses at the $5.15 \mathrm{~W}$ mean laser power and $100 \mathrm{kHz}$ repetition rate.

Another welding test was taken by reducing laser average beam power from $5.15 \mathrm{~W}$ to $1.45 \mathrm{~W}$ by steps of $0.25 \mathrm{~W}$ (or pulse energy from $51.5 \mu \mathrm{J}$ to $14.5 \mu \mathrm{J}$ ) irradiating with 10000 pulses. The crack size in a bead depending on the used pulse energy after welding with irradiation of 10000 pulses is shown in Fig. 8.

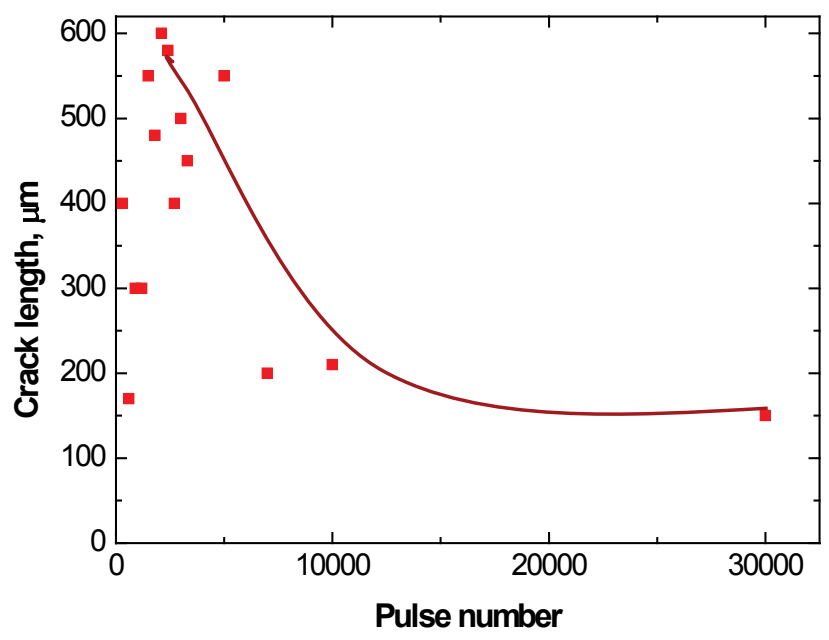

Fig. 7 Crack length in the beads after irradiation at $5.15 \mathrm{~W}$ average beam power and $100 \mathrm{kHz}$ repetition rate. The red line is for eye guidance.

The crack length in the glass bead strongly depended on the laser pulse energy. As it is shown in Fig 8, the cracks in the bead appeared at the pulse energy of $30 \mu \mathrm{J}$ and it started to grow sharply when pulse energy exceeded value of $42.5 \mu \mathrm{J}$. 


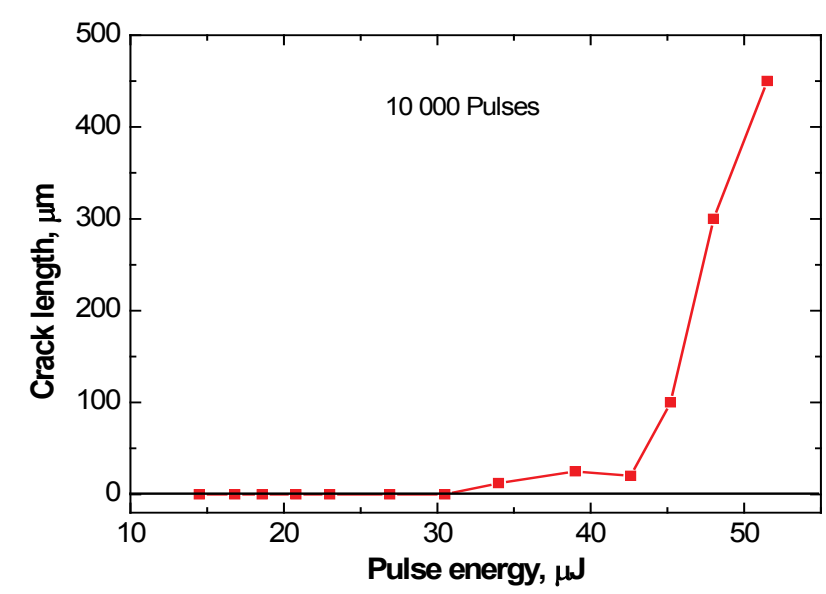

Fig. 8 The crack length versus the pulse energy. The pulse number was 10000 , the pulse repetition rate $-100 \mathrm{kHz}$.

By selecting the laser pulse energy below the crack formation limit, according to Fig. $8(28 \mu \mathrm{J})$, the weld quality was investigated depending on the number of laser pulses. The results are presented in Table 2 .

Table 2 Bead-to-glass welding results. Mean laser power $2.8 \mathrm{~W}$; pulse energy $2.8 \mu \mathrm{J}$, repetition rate $100 \mathrm{kHz}$.

\begin{tabular}{rc}
\hline \# of pulses & result \\
\hline 30 & no weld \\
300 & no weld \\
3,000 & weak weld \\
30,000 & welded \\
300,000 & bead breaks into parts \\
$3,000,000$ & bead breaks into parts \\
\hline
\end{tabular}

Welding of the bead to the substrate was achieved with a few tens thousands of laser pulses. Utilization of excessive laser pulses in this medium pulse energy regime led to the break of the glass beads. The cracks formation and growth during the glass heating by a laser pulse and cooling between pulses are usually initiated by melted glass in the nonlinear absorption region. Expansion of this area causes thermally induced stress which can exceed the glass strength [9]. However, cracks can appear not just close to the melted zone but also in non melted region due to temperature gradient and break propagation.

\subsection{Bead-to-glass welding @ $500 \mathrm{kHz}$}

The glass welding process was investigated with the higher laser pulse repetition rate. No defects in beads were observed even after the higher average laser power of $14.5 \mathrm{~W}$ and the higher number of pulses (1 500000$)$. The cracks did not appear in the welding region after irradiation with the higher pulse repetition rate, because the cooling time between pulses was 5 times shorter than using $100 \mathrm{kHz}$ and thus the transient temperature variation was lower and lower thermal stresses were induced in the glass.

The temperature variation inside the glass is an important issue for the welding. Temperature in the glass during welding is determined by laser parameters: pulse energy, repetition rate, pulse width, number of pulses. Fig. 9 shows the temperature variation with every pulse in the burst for two different pulse repetition rates.

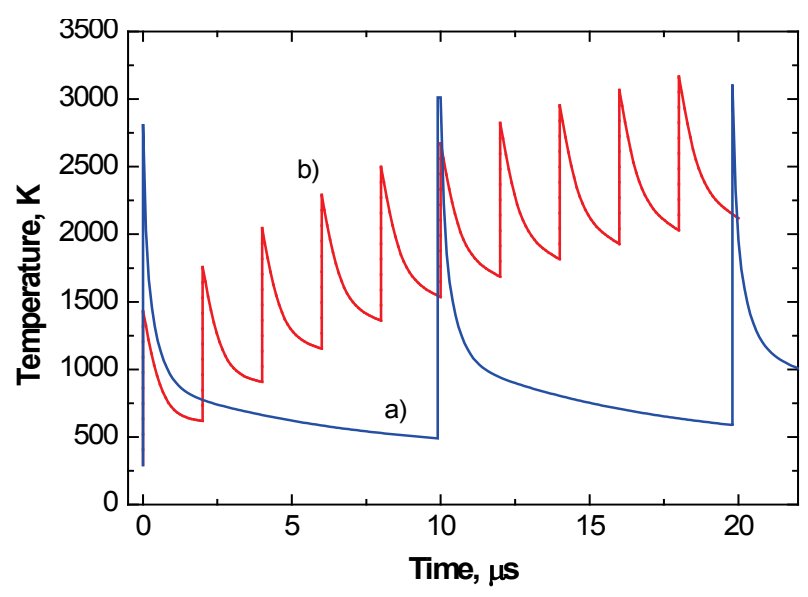

Fig. 9 The thermal cycling in the focal area for the first laser pulses in a welding burst. a) $100 \mathrm{kHz}$ repetition rate; b) $500 \mathrm{kHz}$ repetition rate. The mean laser power $5.15 \mathrm{~W}$.

The high variation in a temperatures is between laser pulses but the background temperature increases with every pulse in the burst, until it stabilizes after more than 10000 pulses, according to simulations in $[7,14]$. At higher temperature glass from a brittle material becomes ductile. This can explain reduction in crack propagation, shown in Fig. 7. In addition the smooth temperature transition from the absorption region to the bulk is also very important for avoiding the cracks. Simulations at $500 \mathrm{kHz}$ shown in Fig. 5 confirm a smooth temperature transition after 30000 applied laser pulses.

Focus position along the optical axis is very important for the welding experiment, because it determinates location of the region of nonlinear absorption inside the sample. The nonlinear absorption strongly depends on the laser irradiation intensity, and thus also on the irradiation power. Increasing of the intensity in the focal region expands the absorption volume. Optimal focus position is related with location of this absorption volume.

Therefore, joining of the glass bead to the substrate was investigated by changing the focal point position along the optical axis relative to the bead-to-glass interface. The irradiation time and average laser power were also adjusted. Test results with $500 \mathrm{kHz}$ irradiation source are presented in Table 3.

Table 3 Bead-to-glass welding results. Mean laser power $2.8 \mathrm{~W}$; pulse energy $2.8 \mu \mathrm{J}$, repetition rate $500 \mathrm{kHz}$.

\begin{tabular}{ccc}
\hline $\begin{array}{c}\text { Optimal } \\
\mathrm{z} \text { displace- } \\
\text { ment }\end{array}$ & Irradiation time & $\begin{array}{c}\text { Average irra- } \\
\text { diation power }\end{array}$ \\
\hline $0.89-0.96 \mathrm{~mm}$ & $0.03-1.5 \mathrm{~s}$ & $5.6-14 \mathrm{~W}$ \\
$0.96-1.0 \mathrm{~mm}$ & $1.5-3 \mathrm{~s}$ & $8-11 \mathrm{~W}$ \\
$0.9-0.93$ & $1.5-3 \mathrm{~s}$ & $5.6-7 \mathrm{~W}$ \\
\hline
\end{tabular}

Here the $\Delta \mathrm{z}$ displacement is used for the focus position change due to the bead focusing. Parametric window here is given just for the glass bead joining to the glass substrate after irradiation. The joint strength of the weld mostly was dependent on the join quality (the crack-free welding) and 
also on the weld seam area. The crack-free joint was possible to achieve at the high pulse repetition rate. The weld area in those experiments depended on the initial glass bead contact with the substrate surface area. The weld area was investigated by increasing irradiation time and the results of the $500 \mathrm{kHz}$ welding with $14 \mathrm{~W}$ average irradiation power are shown in Fig. 10.

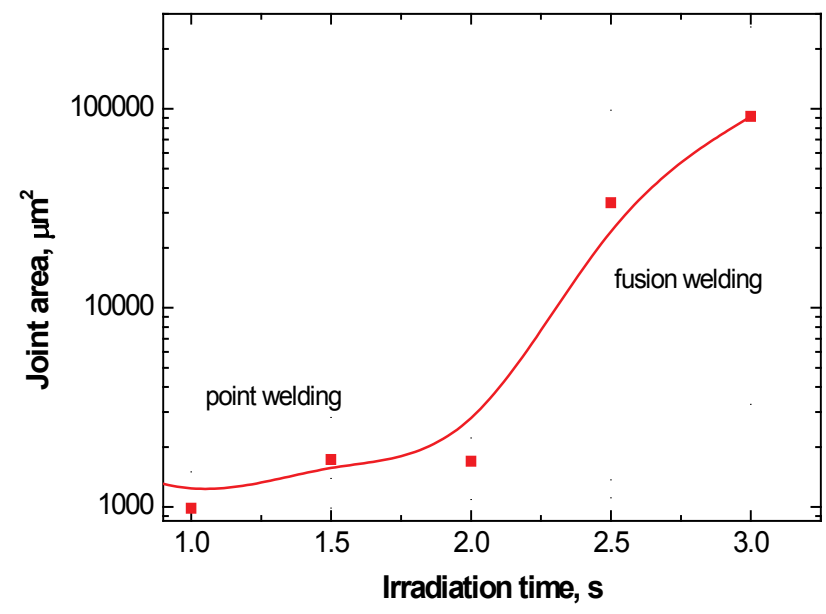

Fig. 10 Welded seam average area dependence on irradiation time. Irradiation power $14 \mathrm{~W}$, repetition rate $500 \mathrm{kHz}$.

For lower irradiation time, the weld area size is similar to the initial contact area between the glass sphere and plate. For the irradiation time higher than 2 seconds, the weld area started to increase rapidly. This wed area growth appeared when a part of the bead was fused and spread over the substrate surface. Here we distinguish two welding regimes: the point welding at the initial contact surface and the welding when a part of the bead is fused on the substrate. The parametric window of both types of welding was limited by the average laser power, irradiation time and focus position.

\section{I type of weld joint- point welding}

The point welding at the contact point between the bead and substrate was achieved in a comparable wide process parameter window, as shown in Table 4.

Table 4 Parameters of the laser point welding.

\begin{tabular}{ccc}
\hline $\begin{array}{c}\text { Focal position, } \\
\mathrm{mm}\end{array}$ & $\begin{array}{c}\text { Irradiation time, } \\
\mathrm{s}\end{array}$ & $\begin{array}{c}\text { Laser power, } \\
\mathrm{W}\end{array}$ \\
\hline $0.88-0.96$ & $0.03-0.3$ & $8-14$ \\
$0.9-1.02$ & $0.5-1.5$ & $5.6-14$ \\
\hline
\end{tabular}

The bead joined by this type of welding is fixed to the substrate and remains on it up-down rotation of the substrate, however the adhesion force is weak and the bead can be easily removed by touching with a piece of paper. Optical image of the weld area is shown in Fig. 11.

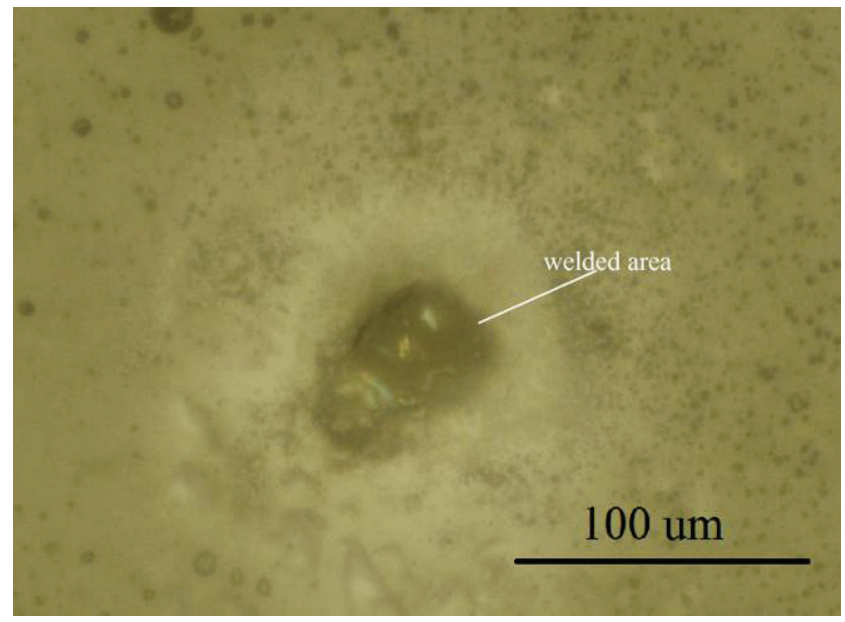

Fig. 11 Optical microscope image of the point weld between the glass bead and plate. Irradiation time $0.5 \mathrm{~s}$, mean laser power $10 \mathrm{~W}$ at the $500 \mathrm{kHz}$ repetition rate.

\section{II type of weld joint - fusion welding}

The fusion weld joint was achieved in significant narrower working window, presented in Table 5. The focal position was controlled with accuracy of $5 \mu \mathrm{m}$.

Table 5 Parameters of the laser fusion welding.

\begin{tabular}{ccc}
\hline $\begin{array}{c}\text { Focal position, } \\
\mathrm{mm}\end{array}$ & $\begin{array}{c}\text { Irradiation time, } \\
\mathrm{S}\end{array}$ & $\begin{array}{c}\text { Laser power, } \\
\mathrm{W}\end{array}$ \\
\hline $0.9 \pm 0.01$ & $2-3$ & $6.5-10$ \\
$0.95-0.97$ & $2-3$ & $9-12.5$ \\
\hline
\end{tabular}

This type of a joint was significant more mechanically rugged, however repeatability of the process was low. Using the same process parameters (laser power, irradiation time, focal position), amount of successfully welded bead was below $30 \%$. The reason could be in deviation from centering of the bead relative to optical axis of the laser beam. The hole' diameter in the focusing head was only $0.11 \mathrm{~mm}$ larger than the mean bead diameter, which permitted misalignment of bead relative laser beam as high as $55 \mu \mathrm{m}$.

Keeping the focus position at the right point ass achieved not only by controlling focus height of the aspheric lens but also the optical axis must coincide well with the bead symmetry axis. Misalignment in the laser beam propagation through the bead leads to coma aberration at the focal plane, which transforms spatial distribution and maximum intensity inside glass. The aberration-introduced distortion not only affects shape of the focus, but also moves its position from the contact point, further in the bead bulk. Due to nonlinear absorption process, these deviations have strong influence of the absorption volume and temperature of the glass.

After longer irradiation time, when the absorption area is far from the contact point, inside the bead bulk, glass is fused in the absorption volume and expands enough to cause the defects - cracks. Differently happens when this absorption area is close to the contact point with the substrate. Expansion of the fused glass is not limited by surrounded infused glass.

Fig. 12 and Fig. 13 show two examples of the fusion welding of beads to glass plate, with good alignment of 
laser beam and the bead axis of symmetry, and when misalignment was a case.

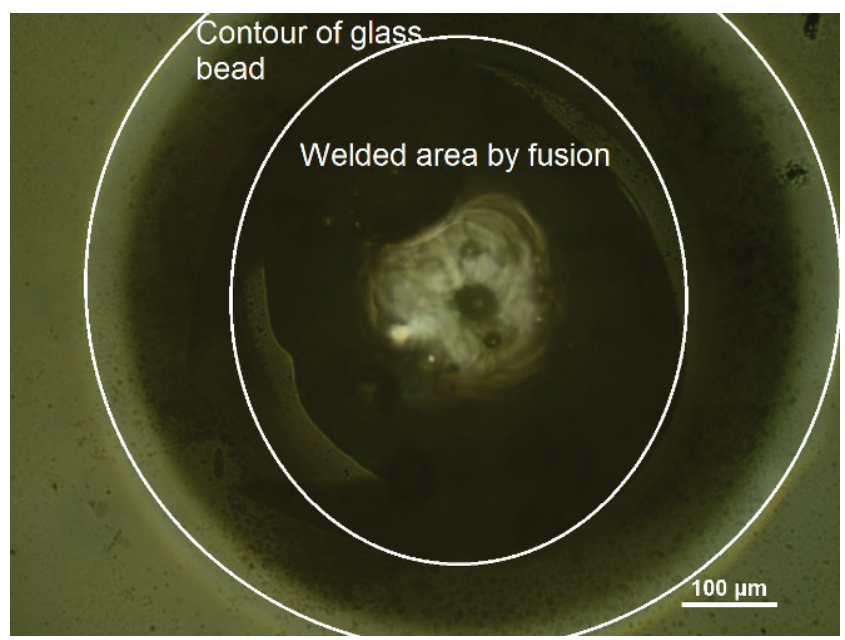

Fig. 12 Optical microscope image of the fusion weld between the glass bead and plate. Irradiation time $3 \mathrm{~s}$, mean laser power $14 \mathrm{~W}$ at the $500 \mathrm{kHz}$ repetition rate.

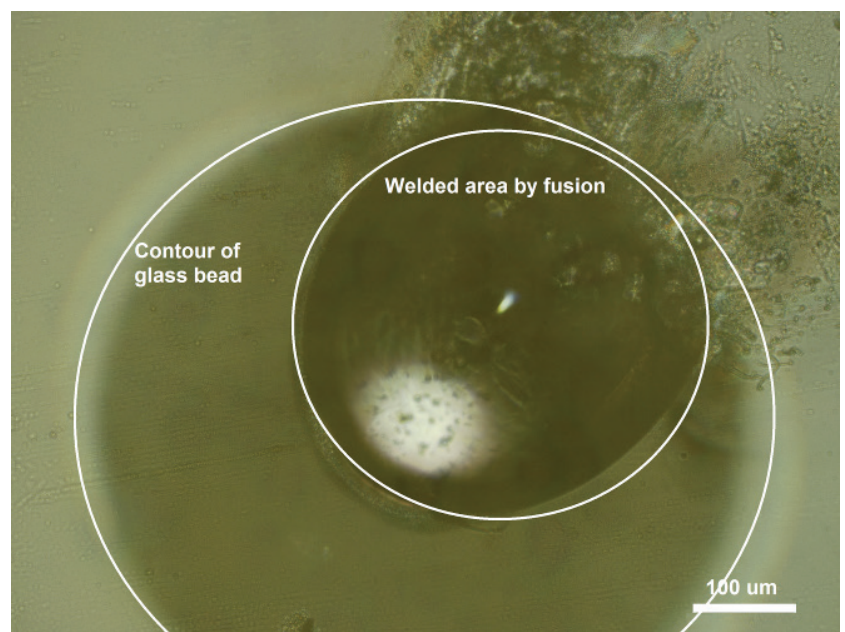

Fig. 13 Optical microscope image of the fusion weld between the glass bead and plate. Irradiation time $2.5 \mathrm{~s}$, mean laser power $14 \mathrm{~W}$ at the $500 \mathrm{kHz}$ repetition rate.

We have studied the influence of the lateral misalignment to the bead welding. The result showed that deviation of optical axis from the symmetry axis of the bead up to $35 \mu \mathrm{m}$ had no affect to the welded joint.

Fig. 14 presents image of the glass plate with beads welded at optimized regime.

\subsection{Testing of the mechanical strength}

In vacuum isolated glass, the stresses due to atmospheric pressure occur in the vicinity of the pillars. Atmospheric pressure produces load of $1 \mathrm{~kg} / \mathrm{cm}^{2}$. To withstand this pressure, spacers should be place at some maximum distance depending on their strength.

A stress test with the beads welded on selected optimal regimes was performed to evaluate the remaining compressive strength of the glass beads due to defects developed during welding process. Cracks could be caused by thermal stresses induced by temperature gradient in region of nonlinear absorption.



Fig. 14 Glass bead welded to the plate.

Compressive strength of the glass beads was investigated depending on the welding regime and compared with the strength of not welded beads. Four beads were welded (or simply placed for comparison) on the glass plate on corners of a virtual rectangular and covered with another glass plate. The force directed downwards was applied to the stack. The load was increased until all beads between the glass plates collapsed. The breakage load was divided by 4 to find the compressive strength value for a single bead. Experiments were repeated 10 times with the beads welded at the same conditions and the resulting mean load to compress the bead for different welding regimes is shown in Table 6.

Table 6 Compressive strength of the glass beads depending on the laser welding regime.

\begin{tabular}{lc}
\hline & Load to compress, $\mathrm{kg} / \mathrm{bead}$ \\
\hline Bead without welding & $13.4 \pm 0.9$ \\
Point welding & $12.3 \pm 4.1$ \\
Fusion welding & $22.8 \pm 4.3$ \\
\hline
\end{tabular}

From the results in Table 6 it is evident that compressive strength of the beads remains nearly the same as of non-welded ones, however the deviation significantly increased, reducing the safety limit. The fusion welding increased the compressive strength of the beads, probably, due to increased contact area with the glass plate

When compressive strength of the glass bead is known from the mechanical strength measurements, it is possible to estimate the quantity of beads per area to withstand atmospheric pressure after evacuation of the glass block. In case of point welding, distance between the glass beads should be no more than $3 \mathrm{~cm}$. The load to a single bead will be as high as $9.18 \mathrm{~kg}$. If fusion welding is applied, the distance can be increased up to $4 \mathrm{~cm}$ with a load of $16 \mathrm{~kg}$ per bead.

\section{Conclusions}

The weld joint of the bead to the glass surface appeared after a certain number of laser pulses.

When repetition rate of $100 \mathrm{kHz}$ was applied for the welding, cracks appeared in the bead and their size depend- 
ed on the laser pulse energy and the number of laser pulses in a burst.

Welding with the higher pulse repetition rate $(500 \mathrm{kHz})$ provides more smooth transient temperature distribution and thermal stresses are reduced significantly, which maintains the crack-free welded area. Welding with excessive melt increased strength of the glass beads.

Defect-free welding of spherical glass beads to a flat glass substrate was achieved with the high repetition rate and low pulse energy irradiation with picosecond laser.

\section{Acknowledgment}

The work was supported by the Agency for Science, Innovation and Technology of the Republic of Lithuania under grant No $31 \mathrm{~V}-13$.

\section{References}

[1] K. Cvecek and I. Miyamoto: J. Laser Micro/Nanoeng., 7 (2012) 68.

[2] P.C. Eames: Vacuum, 82 (2008) 717.

[3] M. Koebel., H. Manz, K. E. Mayerhofer and B. Keller: Solar Energy Materials \& Solar Cells, 94 (2010) 1015.

[4] Y. Arata, H. Maruo, I. Miyamoto and S. Takeuchi: Proc of 7 th International Conf. on Electron and Ion Beam Science and Technology, New Jersey (1976), pp. $111-128$.

[5] I. Miyamoto, A. Horn and J. Gottmann: J. Laser Micro/Nanoeng., 2 (2007) 7.

[6] K. Sugioka, M. Iida, H. Takai and K. Midorikawa: Opt. Lett., 36 (2011) 2734.

[7] I. Miyamoto and K. Cvecek: Physics Procedia, 12 (2011) 378.

[8] K. Cvecek, I. Alexeev, I. Miyamoto and M. Schmidt: Physics Procedia, 5 (2010) 495.

[9] I. Miyamoto, K.Cvecek and M. Schmidt: Opt. Express 19, (2011) 10714.

[10] T. Tamaki, W. Watanabe and K. Itoh: Jpn. J. Appl. Phys., 44 (2005) L687.

[11] C. B. Schaffer, J.F. Garcia and E. Mazur.: Appl. Phys. A 76 (2003) 351.

[12] W. Watanabe Yamada and T. Toma: Appl. Phys. Lett., 89 (2006) 687.

[13] S. Richter, S. Döring and F. Zimmermann: Appl. Phys. A, 103 (2011) 257.

[14] S. Eaton H. Zhang, M. L. Ng, J. Li, W. J. Chen, S. Ho, and P. R. Herman: Opt. Express, 16 (2008) 9443.

(Received: July 5, 2012, Accepted: June 3, 2013) 